\title{
Mostly before Einstein: explanations for the anomalous orbit
}

\section{P.J. Message}

Mercury's Perihelion: From Le Verrier to Einstein. By N.T. Roseveare. Pp.208. ISBN 0-19-858174-2. (Oxford University Press: 1982.) $£ 20, \$ 49$.

INTRODUCTORY accounts of Einstein's general theory of relativity usually place great stress on the importance, amongst observational tests of the theory, of the precession of the orbit of the planet Mercury. Certainly it is a great triumph of that theory that it supplies an explanation of the discrepancy between the observed mean rate of precession of the apse of Mercury and that calculated according to Newton's gravitational theory.

The discrepancy, however, had been known for more than half a century before Einstein's theory was formulated. This book (which is a revision of the author's $\mathrm{PhD}$ thesis) follows through the history of the attempts to explain the anomaly, with a thorough survey of relevant publications and discussion of a number of interesting associated topics.

The explanations which were offered fall into two types: that the discrepancy represents the perturbation of Mercury by hitherto undiscovered matter in the Solar System (usually postulated to lie within the orbit of Mercury); or that it indicates that Newton's law is not a completely accurate description of how gravitation actually acts. The general theory of relativity falls into this second type. The further discrepancy (found by Newcomb) between the observed and predicted rates of precession of the orbit plane of Venus at one time also appeared to be significant, and many of the explanations were formulated so as to account for this too. (General relativity of course does not. It is perhaps strange that debate over this second discrepancy virtually ceased between the time when the apse of Mercury had been adequately dealt with by Einstein's theory, and Duncombe's reanalysis of the observations of Venus, published in 1958, which did not confirm the existence of any discrepancy in its motion.)

The earlier explanations, however, were all of the first type. Since changing the estimates of the masses of the known planets could not remove the discrepancy without producing others elsewhere, undiscovered bodies were postulated. It was soon realized that the hypothetical planet "Vulcan", supposed to move in an orbit within that of Mercury, could not be large enough to have given rise to the necessary perturbation otherwise it would have been certainly seen. Similarly the observed level of brightness of the zodiacal light eventually showed that the matter causing it could not be of sufficient mass to give an adequate explanation of the apparent anomaly in Mercury's orbit.

In the second category of explanation we have "Hall's hypothesis", investigated over some years by Newcomb. Here, the proposed answer was that in the law of gravitation the exponent of the inverse distance is slightly greater than two. Again, this theory was eventually ruled out since it would give effects in the Moon's motion which are not in fact observed. The class of velocity-dependent laws of gravitation, beginning with Weber's, proved equally unable readily to provide the necessary value of the apse motion.

Readers who are confident at mathematical manipulation will no doubt avoid being confused by a number of elementary slips in some of the mathematical explanations given by Roseveare, such as the omission of the largest term in the righthand side of equation (1.11), by the description of the quantities $k_{1}$ and $k_{2}$, in equation (1.12), as "constants" (which they are far from being), and by the use of the notation " $\mathrm{d} w$ " for the angle which is traversed between successive passages through the same apse. Such blemishes, however, need not prevent one's appreciating this otherwise thorough study of the fascinating history with which the book is concerned.

P.J. Message is Reader in Applied Mathematics and Theoretical Physics at the University of Liverpool.

\section{$M$ and $O$ and $S$}

\section{Andrew Holmes-Siedle}

MOS Physics and Technology. By E. Nicollian and J.R. Brews. Pp.924. ISBN 0-471-08500-6. (Wiley: 1982.) f61.95, $\$ 99.10$.

THE technology of metal-oxide-silicon (MOS) structures has provided much of the thrust which has recently given the Information Revolution its breathtaking pace. Hitherto, a problem for the research worker dealing with MOS devices was that few full, connected accounts of the techniques of MOS physics were available to him. The research literature is immense - my own, highly selective files on MOS research occupy over six feet of shelving and the interplay of surface physics, semiconductor physics and electronics is subtle.

Now, at last, Bell Laboratories has come up with the answer and produced another of its gospels. A few months ago, reviewing a textbook emanating from the same establishment, I noted the unique aura of work done there. Nicollian and Brews have produced a book with that same aura - a compound of authority and apparently effortless ease in dealing with a complex, interdisciplinary subject.

Because their aim was to provide unambiguous statements concerning the three layers - metal, oxide, semiconductor - and the crucial interfacial regions between them, the authors have concentrated attention on the simplest possible MOS structure, the MOS capacitor. At the same time, they do not ignore the implications for or needs of the designer of silicon integrated circuits, small chips which include thousands of MOS structures, interwoven with doped (usually diffused) regions. They do, however, omit mention of other semiconductors such as the increasingly important III-V series, probably because MOS technology is not as well-established for these materials.

The MOS capacitor structure in silicon consists of a dot of metal lying on the surface of a silicon dioxide layer, such a layer usually having been formed by thermal oxidation of the underlying silicon in dry oxygen, steam or some similar ambient. The properties of the oxide are extremely sensitive to the method of growth and it might be said that, in the field of high technology, few interacting systems of solids have been as well characterized as the MOS system. This is because of the strong impact which variations in physical structure and purity have on the final electrical characteristics of the working solid-state circuit.

The main tool for investigating those characteristics is the capacitance-voltage plot. As the authors demonstrate, the $\mathrm{C}-\mathrm{V}$ curve, which can be plotted quickly and automatically, is pregnant with information. Various electrical stresses or other excitations such as electron beams can strongly affect this plot. In separate chapters, the authors describe how to extract from $\mathrm{C}-\mathrm{V}$ measurements such details as the nature of the interfacial charge traps, interfacial nonuniformities, the doping and carrier lifetime in the silicon and the important and diverse range of network defects which can populate the glassy oxide layer. The versatility and usefulness of the MOS capacitance and conductance techniques are fully worked out and practical experimental details, such as circuits which have not been widely published before, are given. It is thus not surprising that the book is the size of a small bible; for one community of zealots, its international authority may also approach the biblical.

Andrew Holmes-Siedle is Consultant in Solid State Physics and Radiation Physics to the Fulmer Research Institute, Stoke Poges, Buckinghamshire. 